\title{
Using Steady-State Suppression to Improve Speech Intelligibility in Reverberant Environments for Elderly Listeners
}

\author{
Takayuki Arai, Senior Member, IEEE, Nao Hodoshima, Member, IEEE, and Keiichi Yasu, Member, IEEE
}

\begin{abstract}
Reverberation is a large problem for speech communication and it is known that strong reverberation affects speech intelligibility. This is especially true for people with hearing impairments and/or elderly people. Several approaches have been proposed and discussed to improve speech intelligibility degraded by reverberation. Steady-state suppression is one such approach, in which speech signals are processed before being radiated through the loudspeakers of a public address system to reduce overlap masking, which is one of the major causes of degradation in speech intelligibility. We investigated whether the steady-state suppression technique improves the intelligibility of speech in reverberant environments for elderly listeners. In both simulated and actual reverberant environments, elderly listeners performed worse than younger listeners. The performance in an actual hall was better than with simulated reverberation, and this was consistent with the results for younger listeners. Although the normal hearing group performed better than the presbycusis group, the steady-state suppression technique improved the intelligibility of speech for elderly listeners as was observed for younger listeners in both simulated and actual reverberant environments.
\end{abstract}

Index Terms-elderly people, reverberation, speech intelligibility, steady-state suppression.

\section{INTRODUCTION}

$\mathbf{R}$ EVERBERATION is a large problem that must be overcome to build a "barrier-free" environment for speech communication. It is known that strong reverberation affects speech intelligibility. Although early reflections often help speech intelligibility (the Haas effect, e.g., [1]), late reflections degrade speech intelligibility (e.g., [2]). This is especially true for people with hearing impairments and/or elderly people [3], as well as for non-native listeners [4]. Therefore, designing acoustics of a room becomes increasingly important for creating a universally accessible environment for speech communication.

Manuscript received October 12, 2009; revised May 09, 2010. Date of current version August 13, 2010. This work was supported in part by the Sophia University Open Research Center from MEXT. This work was supported in part the by Sophia University Open Research Center from MEXT. The associate editor coordinating the review of this manuscript and approving it for publication was Dr. Masato Miyoshi.

T. Arai is with the Department of Information and Communication Sciences, Sophia University, Tokyo 102-8554 Japan (e-mail: arai@sophia.ac.jp).

N. Hodoshima is with the Department of Information Media Technology, Tokai University, Tokyo 108-8619 Japan (e-mail: hodoshima@ tokai-u.jp).

$\mathrm{K}$. Yasu is with the Graduate School of Science and Technology, Sophia University, Tokyo 102-8554 Japan (e-mail: k-yasu@ @ophia.ac.jp).

Color versions of one or more of the figures in this paper are available online at http://ieeexplore.ieee.org.

Digital Object Identifier 10.1109/TASL.2010.2052165

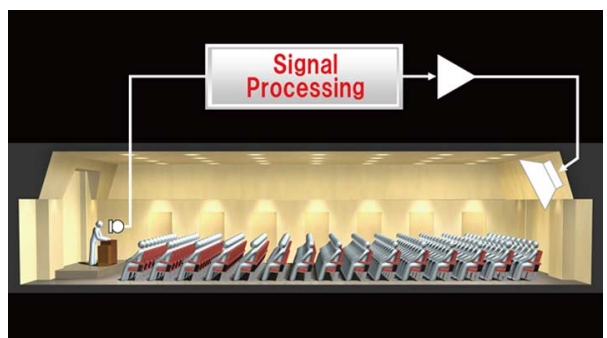

Fig. 1. Steady-state suppression in the public address to improve speech intelligibility.

It has been pointed out that overlap masking in reverberant environments is one of the main sources of degradation in speech intelligibility [5]-[7]. Overlap masking occurs among segments where the reverberation tails of the previous segments mask the following ones. As a result, speech segments following reverberating segments are more difficult to understand. The effect of overlap masking is greater when a previous segment is more powerful than a following segment, as in the case of a vowel followed by a consonant [8], [9].

Several approaches have been proposed and discussed to improve speech intelligibility degraded by reverberation. Some are based on architectural acoustics and others on electrical acoustics. In approaches based on electrical acoustics, there are two major possibilities: having a device near the listener, or having a device in the public address (PA) system (Fig. 1). In the latter case, Arai et al. [8], [9] proposed "steady-state suppression," in which speech signals are processed before being radiated through the loudspeakers of a PA system to reduce overlap masking.

Steady-state suppression [8], [9] is a technique that directly reduces overlap masking caused by reverberation and prevents the degradation of speech intelligibility in a reverberant environment. Strange et al. [10] showed that the information in steady-state portions of a speech signal was relatively insignificant compared with the information in transient portions. Additionally, steady-state portions usually have more energy compared to transients. The steady-state suppression technique reduces overlap masking by estimating and suppressing the more powerful yet less significant steady-state portions of speech, such as the nuclei of syllables. (Here, the term "suppression" is used to refer to simple attenuation of the amplitudes of the steady-state portions.) From the results of several experiments, we have already confirmed its effectiveness when we apply this processing between a microphone and loudspeaker. 
Hodoshima et al. [11], [12] confirmed that it significantly improves speech intelligibility for young normal-hearing listeners in simulated and actual sound fields (reverberation times of $0.8-1.3 \mathrm{~s})$

Not many studies, however, have been done to increase the intelligibility of speech for elderly listeners. Nábělek and Donahue [13] tested assistive listening systems (frequency modulation of radio frequencies and modulation of infrared light waves as transmission modes) in a reverberant environment compared with a PA system, and found that the former yielded higher word identification scores for elderly listeners. Gordon-Salant [14], [15] reported that increasing the energy of consonants relative to that of vowels yielded a higher consonant recognition rate in noise for elderly listeners. Hodoshima et al. [16] reported that the steady-state suppression technique improved the speech intelligibility of a single elderly listener with presbycusis in simulated sound fields (reverberation times of $0.7,1.0$, and $1.2 \mathrm{~s}$ ).

In this paper, we tested whether elderly listeners perform worse in terms of the intelligibility of speech with reverberation than younger listeners due to spectral and/or temporal deficits, and we further tested whether the steady-state suppression technique helps to improve speech intelligibility for elderly listeners as has been observed for younger listeners. We conducted two experiments. In Experiment 1 [17], we tested how elderly listeners perform with regard to the intelligibility of speech with stimuli similar to those used in a previous study for young normal-hearing listeners [12]. In this experiment, the listening tests were conducted in simulation by convolving speech signals with impulse responses. In Experiment 2, we tested elderly listeners' performance in an actual hall. Finally, we compared the elderly listeners' performance in two sound fields, and we further discussed whether avoiding suppression of the steady-state consonants affects the results. In Sections II and III, the two listening tests are described. We further discuss the issues with reference to our experimental results in Section IV.

\section{EXPERIMENTS}

\section{A. Participants}

Experiment 1: Forty-nine elderly listeners (21 males and 28 females, aged 56 to 90 years, average age of 67.9 years) participated in Experiment 1. All were native speakers of Japanese. We divided the participants into three groups based on their audiograms: the normal hearing group (ENH group, 20 participants), the presbycusis group (PRE group, 16 participants) with gradually sloping hearing loss, and the other hearing-loss group (13 participants), which was made up of subjects who had hearing loss not classified as presbycusis. Figs. 2 and 3 show the audiograms of the ENH and PRE groups, respectively. The definition of normal hearing in this study was that pure tone thresholds were less than 25-dB HL at frequencies between 125 and $8000 \mathrm{~Hz}$.

Experiment 2: Twenty-three elderly listeners (6 males and 17 females, aged 64 to 92 years, average age of 72.2 years) participated in Experiment 2. All were native speakers of Japanese. We divided the participants into three groups based on their audiograms: the ENH group (8 participants), the PRE group (3

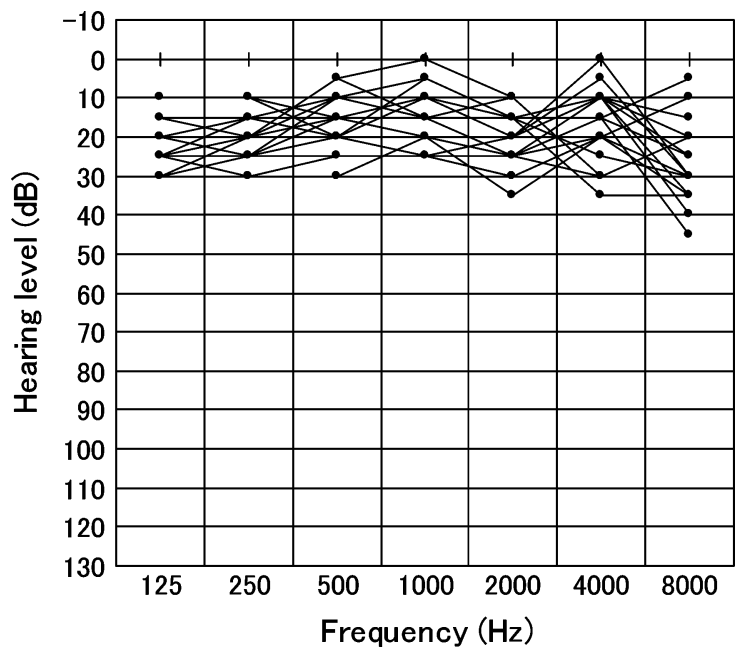

Fig. 2. Audiograms of the normal hearing (ENH) group (20 listeners) in Experiment 1 (some data are overlaid).

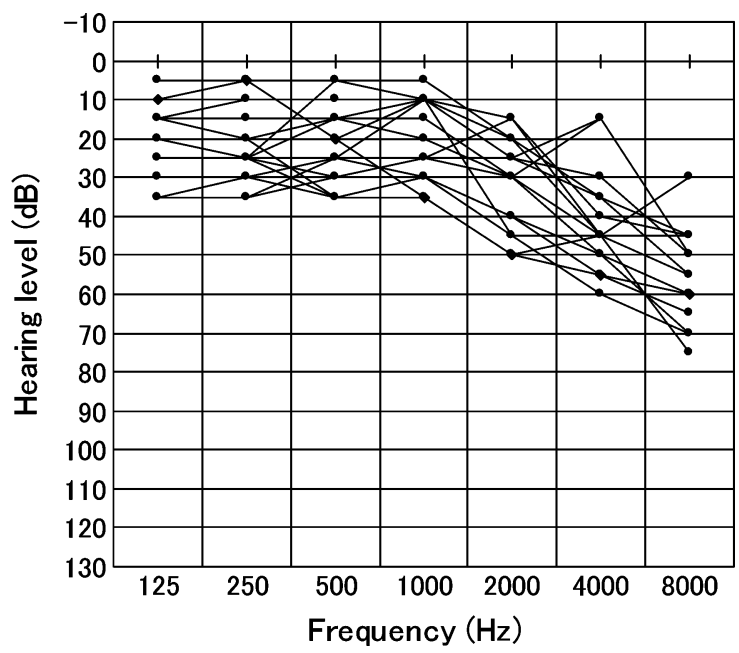

Fig. 3. Audiograms of the presbycusis (PRE) group (16 listeners) in Experiment 1 (some data are overlaid).

participants), and the other hearing-loss group (12 participants), which was made up of subjects who had hearing loss not classified as presbycusis.

\section{B. Steady-State Suppression}

In both experiments, we adopted the same algorithm for the steady-state suppression method as used in previous studies [8], [9], [11], [12]. This technique first splits an original signal (sampled at $16 \mathrm{kHz}$, in this study) into $1 / 3$-octave bands by bandpass filters (BPFs) approximating critical bands. Then, it extracts the temporal envelope in each band by the Hilbert transform. After low-pass filtering at a cutoff frequency of $35 \mathrm{~Hz}$ and down-sampling with a factor of 160, the regression coefficients are calculated from the five adjacent values of the time trajectory of the logarithmic envelope of each band. Then the mean square for the regression coefficients over all bands, $D$, is calculated every $10 \mathrm{~ms}$. This parameter $D$ is similar to what Furui proposed to measure spectral transition [18]. After up-sampling to the original sampling rate, we define a portion of speech as steady-state when $D$ is less than a given threshold (that is the median in this 
(a)

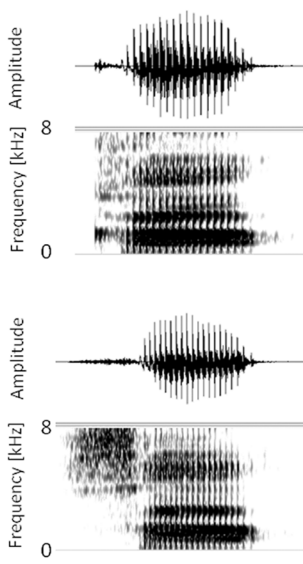

(b)

(c)

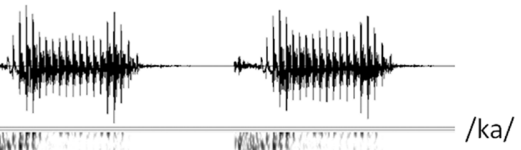

$/ \mathrm{ka} /$
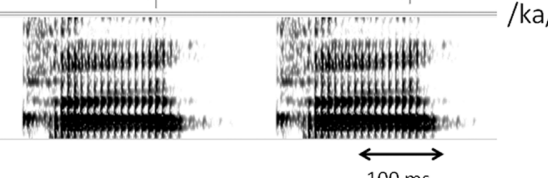

$\longleftrightarrow 100 \mathrm{~ms}$

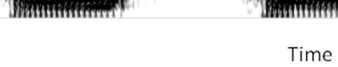

Time
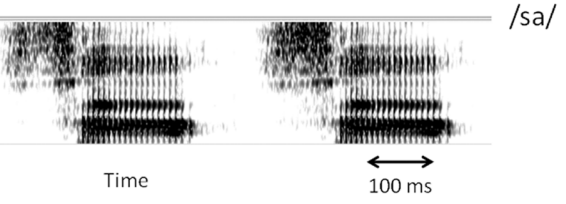

Fig. 4. Original and processed speech signals with the steady-state suppression. The top panel shows the waveforms and their spectrograms of the Japanese syllable $/ \mathrm{ka} /$, and the bottom panel shows them for $/ \mathrm{sa} /$. (a) The original signals, (b) the processed signals with the steady-state suppression, and (c) the processed signals with the steady-state suppression and the additional step.

study). Once a speech portion is considered steady state, the amplitude of the portion is suppressed. In this paper, the speech portion is suppressed to $40 \%$ of the original amplitude, as in previous studies [8], [9], [11], [12].

In Experiment 2, we further added a step to avoid suppressing relatively longer continuants where the spectral moment is higher than $3750 \mathrm{~Hz}$ [19], [20], such as sibilant consonants.

Fig. 4 shows the original and the processed speech signals with the steady-state suppression. The top panel shows the waveforms and their spectrograms of the Japanese syllable $/ \mathrm{ka} /$, and the bottom panel shows them for $/ \mathrm{sa} /$. Fig. 4(a) is the original signals, (b) is the processed signals with the steady-state suppression, and (c) is the processed signals with the steady-state suppression and the additional step. As shown in Fig. 4(b), the steady-state portions of the vowel /a/ are attenuated for both $/ \mathrm{ka} /$ and $/ \mathrm{sa} /$. In addition, the relatively steady portion of /s/ in Fig. 4(b) is attenuated, whereas $/ \mathrm{k} /$ is unchanged. In contrast, the consonants $/ \mathrm{k} /$ and $/ \mathrm{s} /$ are not changed in Fig. 4(c), because the additional step avoids attenuating steady portions in sibilants.

\section{Stimuli}

Experiment 1: The original speech samples consisted of 14 nonsense consonant-vowel (CV) syllables embedded in a Japanese carrier phrase, "Daimoku to shite wa _ to iimasu" (It is called _ as a title). The vowel was $/ \alpha /$ and the consonants were $/ \pi, \tau, \kappa, \beta, \delta, \gamma, \sigma, \Sigma, \eta, \delta \zeta, \delta Z, \tau \Sigma, \mu, \nu /$. The speech samples were originally obtained from the ATR Speech Database of Japanese. The CV syllables were obtained from the monosyllable data set, whereas the carrier phrase was a combination of two partial sentences obtained from the sentence data set. The ratio of the root-mean square (RMS) in the carrier phrase to that in the CVs was adjusted to 1:0.7.
In Experiment 1, we conducted a perceptual experiment under artificial reverberant environments achieved by convolving speech samples with impulse responses. We prepared original speech samples (Proc0) and speech samples processed by steady-state suppression (Proc1). All speech samples were convolved with each of the two impulse responses: Rev1 and Rev2. The reverberation times (T60s) of the two impulse responses we used were $1.3 \mathrm{~s}(\operatorname{Rev} 1)$ and $1.0 \mathrm{~s}(\operatorname{Rev} 2)$. The former impulse responses were obtained at the largest lecture hall at Sophia University in Tokyo. The latter one was originally obtained at Hamming Hall in Tokyo (T60 was $1.1 \mathrm{~s}$ when a reflection board was not used), and we modified its T60 to 1.0 $\mathrm{s}$ by multiplying an exponential decay as in previous studies (e.g., [12]).

Experiment 2: The original speech samples were essentially the same as in Experiment 1, except that the vowels were $/ \alpha /$ and $/ \mathrm{l}$. All possible $\mathrm{CV}$ combinations were selected, excluding those that do not meet Japanese phonotactics. We prepared original speech samples (Proc0) and processed speech samples by two types of steady-state suppression (Proc1 and Proc2), where Proc1 was the original method of steady-state suppression as used in Experiment 1, while Proc2 was the modified method with an additional step to avoid suppressing relatively longer continuants where the spectral moment is higher than $3750 \mathrm{~Hz}$.

\section{Procedures}

Experiment 1: The listening test was conducted in a soundtreated room for each participant one at a time. Stimuli were presented diotically through headphones (STAX SR-303) connected to a computer. The sound level was adjusted to each listener's comfort level during the training session prior to the listening test and kept constant. A stimulus was presented once in each trial and the listeners were instructed to write which of the 14 provided CVs they heard in kana orthography on an answer sheet. After they wrote a response, the next stimulus was presented by clicking a "Next" button on the screen with a mouse. The listening test was carried out at each listener's own pace. For each listener, 56 stimuli (2 processing conditions $\times 14 \mathrm{CVs} \times 2$ reverberation conditions) were presented randomly.

Experiment 2: In Experiment 2, we conducted a listening test in an actual hall: the largest lecture hall in Sophia University. The volume was approximately $3100 \mathrm{~m}^{3}$; the seating capacity was 822 seats. All 23 participants were tested in groups; they were seated every two seats in the center back area of the hall. The stimuli were played through loudspeakers (Electro-Voice TS-9040D-LX) mounted at the center ceiling of the hall. Although a signal-to-noise ratio (SNR) was not measured during the listening test, the SNR should have been high enough to prevent distraction because all equipment in the lecture hall (i.e., a projector and an air ventilation system) was turned off except for the public address system and the computer used for the listening test. The distance between the participants and the loudspeakers was more than $10 \mathrm{~m}$, and this was longer than the critical distance of this hall (approximately $9.8 \mathrm{~m}$ ), so that the reverberant sounds would be more dominant than the direct sound. The sound level was adjusted to participants' comfort level during the training session prior to the listening test and kept constant. All 72 stimuli (3 processing conditions $\times 24$ 

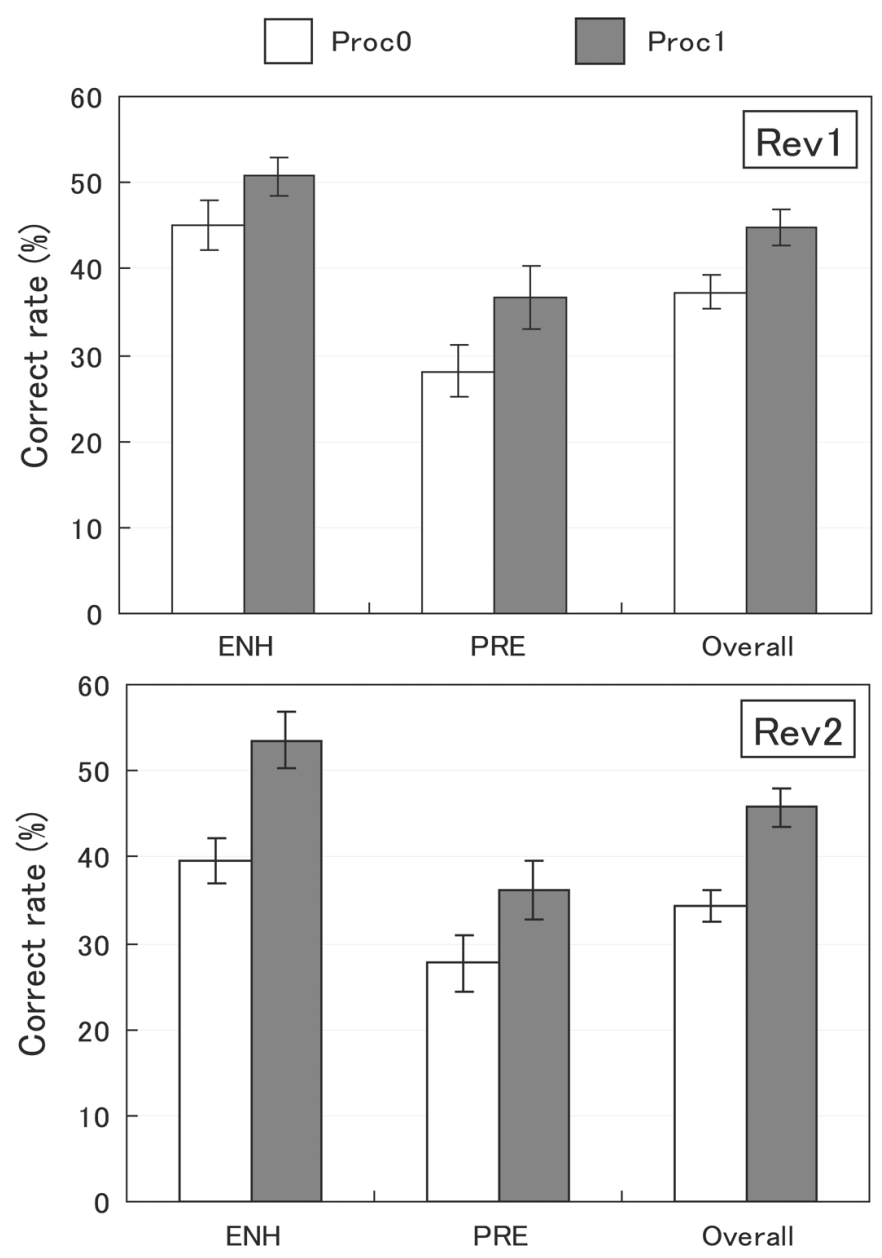

Fig. 5. Correct rates for Proc0 and Proc1 of the ENH group, the PRE group, and overall in Experiment 1. The top is with Rev1 and the bottom is with Rev2. Error bars show standard errors.

CVs) were presented randomly. A stimulus was presented once in each trial and the listeners were instructed to write which of the 24 provided CVs they heard in kana orthography.

\section{EXPERIMENTAL RESULTS}

\section{A. Experiment 1}

Fig. 5 shows the results of Experiment 1 [17]. The bold solid and bold dashed lines show the overall mean percent correct of all participants. A $2 \times 2$ ANOVA was carried out with reverberation (Rev1, Rev2) and processing (Proc0, Proc1) as repeated variables and the correct rate (raw data) as the dependent variable. Results showed that processed speech had significantly higher correct rates than unprocessed speech $(p<0.01)$. A $t$-test showed that processed speech had significantly higher correct rates than unprocessed speech in both reverberant environments $(p<0.01)$. The main effect of reverberation, as well as the interaction between reverberation and processing, was not significant.

In both the ENH and PRE groups, a $2 \times 2$ ANOVA was carried out with reverberation (Rev1, Rev2) and processing (Proc0, Proc1) as repeated variables, and the correct rate as the dependent variable. Results showed that processed speech had significantly higher correct rates than unprocessed speech for both

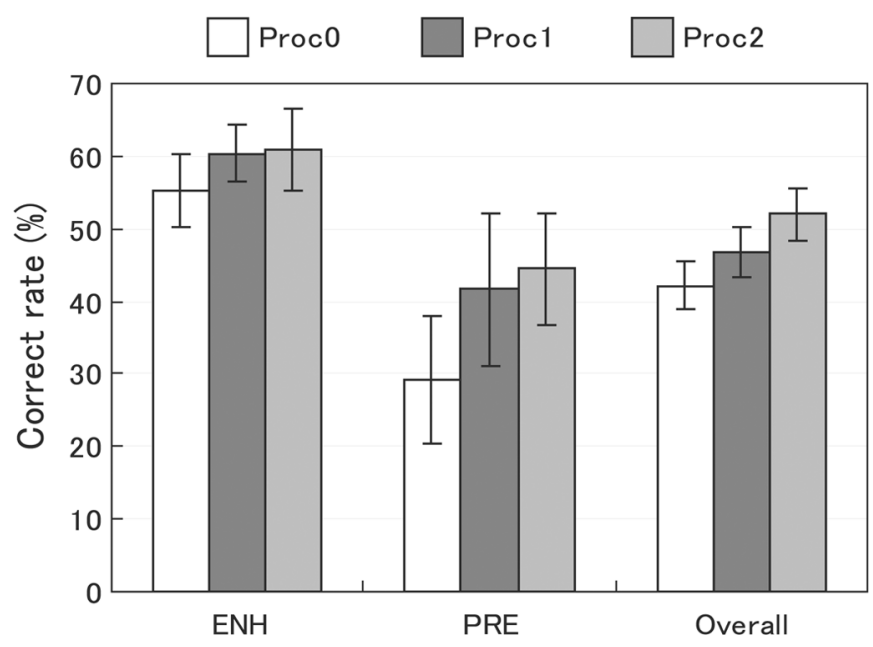

Fig. 6. Correct rates for Proc0, Proc1, and Proc2 of the ENH group, the PRE group, and overall in Experiment 2. Error bars show standard errors.

groups $(p<0.01)$. A $t$-test showed that processed speech had significantly higher correct rates than unprocessed speech in both reverberant environments for both groups (ENH: Rev1: $p=0.05, \operatorname{Rev} 2: p<0.01$; PRE: Rev1: $p=0.03$, Rev2: $p<0.01)$. The main effect of reverberation was not significant for either group. The interaction between reverberation and processing was significant for the ENH group $(p<0.02)$, but not for the PRE group.

\section{B. Experiment 2}

Fig. 6 shows the results of Experiment 2. A $3 \times 1$ ANOVA was carried out with processing (Proc0, Proc1, Proc2) as repeated variables and the correct rate (raw data) as the dependent variable. Results showed that the main effect of processing was statistically significant $(p<0.01)$. A Sidak multiple comparison test showed that Proc2 had significantly higher correct rates than Proc0 and Proc1 [Proc0: $p<0.01$, Proc1: $p=0.03$ ], showing that the steady-state suppression also significantly improved the intelligibility of speech in an actual hall.

\section{DISCUSSION}

Results of Experiment 1 showed that the steady-state suppression was also effective for elderly listeners, including both those with normal hearing and presbycusis, as well as for young listeners (e.g., [12]). Fig. 7 shows the overall mean percent correct in Experiment 1 and a previous study of young normal hearing listeners [11], [12] in Rev1 and Rev2 (the correct rates of young people in Higashi-Yamato hall were recalculated from [12], in which the target vowel was /a/, while the [12] used /a, i/ as the targets). In the previous study, the correct rate of the processed stimuli was also significantly higher than that of the unprocessed stimuli in each reverberant environment. The fact that the mean correct rates of the elderly listeners in Experiment 1 were lower than those of the young normal-hearing listeners in Hodoshima et al. [11], [12] may support the studies of Gordon-Salant and Fitzgibbons [21], [22], which reported that audibility and auditory temporal resolution degrade with age and therefore elderly listeners become greatly affected by reverberation. Although the young normal-hearing listeners showed higher correct rates, 


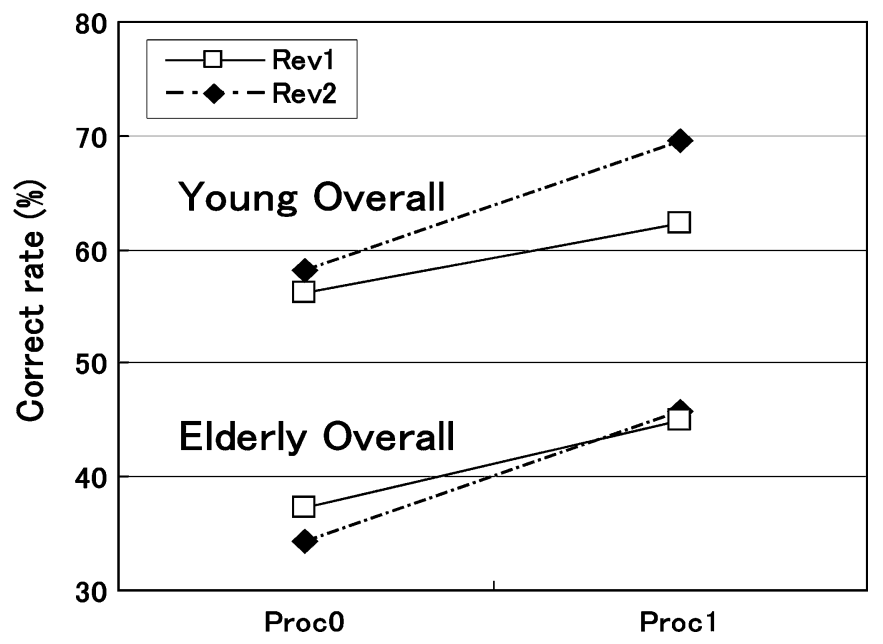

Fig. 7. Correct rates for Proc0 and Proc1 of the elderly listeners (Experiment 1) and of the young normal-hearing listeners [11], [12].

the improvements in speech intelligibility by steady-state suppression were similar for both listener groups. Therefore, the improvement rates (the improvements divided by the correct rates) are higher for the elderly group. This may suggest that steady-state suppression contributes not only to reducing the overlap masking but also to reducing the temporal masking that was increased due to the poor temporal resolution of elderly listeners.

Results of Experiment 2 showed that the steady-state suppression was also effective for elderly listeners in an actual hall as well as in the simulated reverberant environment (Experiment 1). Because the impulse response data used as Rev1 in Experiment 1 was obtained from the same hall used in Experiment 2, we can compare the results of Proc0/Proc1 in Rev1 of Experiment 1 and Proc0/Proc 1 of Experiment 2 to see the effect of the different listening conditions: diotically simulated reverberation and natural reverberation in an actual hall. Comparing the results shows that those in an actual hall were higher (Proc0: 42.2\%; Proc1: 46.7\%) than those in the diotically simulated reverberation (Proc0: 37.3\%; Proc1: $44.9 \%$ ). This tendency is similar to the results reported for young normal-hearing listeners in Hodoshima et al. [11]. It is also consistent with Helfer [23] and may be caused by binaural advantage [24], although the experimental setups were not identical (the participants were tested one at a time in Exp. 1 but tested in groups in Exp. 2) and these differences might have caused the different performance.

In Experiment 2, the mean correct rate of Proc2 was significantly higher $(52.0 \%)$ than that of Proc1 $(46.7 \%)(p<0.05)$. The medial portion of the continuants, such as $/ \alpha /$ and $/ \Sigma /$, was suppressed in Proc1 but not in Proc2, and this seems to have yielded the difference in performance for Proc0 and Proc1. The mean correct rates of these two continuants were 59.4, 49.3, and $71.0 \%$ for Proc0, Proc1, and Proc2, respectively. While suppressing the continuants did not affect the performance for young normal-hearing listeners in previous studies, it affected the elderly listeners' performance drastically. In both experiments, we used the listener's comfort level. It is known that elderly listeners tend to set their comfort level below the optimum presentation level. Additionally, sibilants were weak in
Proc 1, and this might have lead to particularly poor performance for the elderly listeners with Proc1. Although this is the case, in summary, preserving steady-state portions of consonants helped elderly listeners to achieve higher speech intelligibility.

\section{CONCLUSION}

We investigated whether the steady-state suppression technique improves the intelligibility of speech in reverberant environments for elderly listeners. In both simulated and actual reverberant environments, elderly listeners performed worse than younger listeners, due possibly to temporal deficit. Furthermore, the steady-state suppression technique improved the intelligibility of speech for elderly listeners as has been observed for younger listeners (e.g., [12]). The performance in an actual hall was better than in simulated reverberation, and this tendency was consistent with the results for younger listeners (e.g., [12]). The normal hearing group performed better than the presbycusis group. As a next step, in future work we will test whether we can obtain a similar tendency for different speech materials, such as words and sentences.

\section{ACKNOWLEDGMENT}

The authors would like to thank H. Tachibana, K. Ueno, and S. Yokoyama for providing the impulse response data. They would also like to thank Y. Miyauchi, K. Kobayashi, K. Kurisu, and M. Shindo for their kind support.

\section{REFERENCES}

[1] H. Haas, "The influence of a single echo on the audibility of speech," J. Audio Eng. Soc., vol. 20, pp. 145-159, 1972.

[2] A. K. Nábělek and J. M. Pickett, "Monaural and binaural speech perception through hearing aids under noise and reverberation with normal and hearing-impaired listeners," J. Speech Hear. Res., vol. 17, pp. 724-739, 1974.

[3] A. K. Nábělek and D. Mason, "Effect of noise and reverberation on binaural and monaural word identification by subjects with various audiograms," J. Speech Hear. Res., vol. 24, pp. 375-383, 1981.

[4] Y. Takata and A. K. Nábělek, "English consonant recognition in noise and in reverberation by Japanese and American listeners," J. Acoust. Soc. Amer., vol. 88, no. 2, pp. 663-666, 1990.

[5] V. O. Knudsen, "The hearing of speech in auditoriums," J. Acoust. Soc. Amer., vol. 1, no. 1, pp. 56-82, 1929.

[6] R. H. Bolt and A. D. MacDonald, "Theory of speech masking by reverberation," J. Acoust. Soc. Amer., vol. 21, no. 6, pp. 577-580, 1949.

[7] A. K. Nábělek, T. R. Letowski, and F. M. Tucker, "Reverberant overlap- and self-masking in consonant identification," J. Acoust. Soc. Amer., vol. 86, no. 4, pp. 1259-1265, 1989.

[8] T. Arai, K. Kinoshita, N. Hodoshima, A. Kusumoto, and T. Kitamura, "Effects of suppressing steady-state portions of speech on intelligibility in reverberant environments," in Proc. Autumn Meet. Acoust. Soc. Jpn., 2001, vol. 1, pp. 449-450, (in Japanese).

[9] T. Arai, K. Kinoshita, N. Hodoshima, A. Kusumoto, and T. Kitamura, "Effects on suppressing steady-state portions of speech on intelligibility in reverberant environments," Acoust. Sci. Tech., vol. 23, no. 4, pp. 229-232, 2002.

[10] W. Strange, J. J. Jenkins, and T. L. Johnson, "Dynamic specification of coarticulated vowels," J. Acoust. Soc. Amer., vol. 74, no. 3, pp. 695-705, 1983

[11] N. Hodoshima, T. Goto, N. Ohata, T. Inoue, and T. Arai, "The effect of pre-processing approach for improving speech intelligibility in a hall: Comparison between diotic and dichotic listening conditions," Acoust. Sci. Tech., vol. 26, no. 2, pp. 212-214, 2005.

[12] N. Hodoshima, T. Arai, A. Kusumoto, and K. Kinoshita, "Improving syllable identification by a preprocessing method reducing overlapmasking in reverberant environments," J. Acoust. Soc. Amer., vol. 119, no. 6 , pp. 4055-4064, 2006. 
[13] A. K. Nábělek and A. M. Donahue, "Comparison of amplification systems in an auditorium," J. Acoust. Soc. Amer., vol. 79, no. 6, pp. 2078-2082, 1986.

[14] S. Gordon-Salant, "Recognition of natural and time/intensity altered CVs by young and elderly subjects with normal hearing," J. Acoust. Soc. Amer., vol. 80, no. 6, pp. 1599-1607, 1986.

[15] S. Gordon-Salant, "Effects of acoustic modification on consonant recognition by elderly hearing-impaired subjects," J. Acoust. Soc. Amer., vol. 81, no. 4, pp. 1199-1202, 1987.

[16] N. Hodoshima, Y. Miyauchi, K. Yasu, and T. Arai, "Steady-state suppression for improving syllable identification in reverberant environments: A case study in an elderly person," Acoust. Sci. Tech., vol. 28, no. 1, pp. 53-55, 2007.

[17] Y. Miyauchi, N. Hodoshima, K. Yasu, N. Hayashi, T. Arai, and M. Shindo, "A preprocessing technique for improving speech intelligibility in reverberant environments: The effect of steady-state suppression on elderly people.," Proc. Interspeech, pp. 2769-2772, 2005.

[18] S. Furui, "On the role of spectral transition for speech perception," $J$. Acoust. Soc. Amer., vol. 80, no. 4, pp. 1016-1025, 1986.

[19] K. Kobayashi, Y. Hatta, K. Yasu, S. Minamihata, N. Hodoshima, T. Arai, and M. Shindo, "Improving speech intelligibility for elderly listeners by steady-state suppression," Tech. Rep. IEICE Jpn., vol. SP2005-168, pp. 31-36, 2006, (in Japanese).

[20] K. Kobayashi, K. Yasu, N. Hodoshima, T. Arai, and M. Shindo, "A study of syllable enhancement for elderly listeners by suppression energy of steady-state portions of vowels," J. Acoust. Soc. Jpn., vol. 64, no. 5, pp. 278-289, 2008, (in Japanese).

[21] S. Gordon-Salant and P. J. Fitzgibbons, "Profile of auditory temporal processing in older listeners," J. Speech, Lang., Hear. Res., vol. 42, pp. 300-311, 1999.

[22] P. J. Fitzgibbons and S. Gordon-Salant, "Age effects on discrimination of timing in auditory sequences," J. Acoust. Soc. Amer., vol. 116, no. 2, pp. 1126-1134, 2004.

[23] K. S. Helfer, "Binaural cues and consonant perception in reverberation and noise," J. Speech Hear. Res., vol. 37, pp. 429-438, 1994.

[24] W. Koenig, "Subjective effects on binaural hearing," J. Acoust. Soc. Amer., vol. 22, pp. 61-62, 1950.

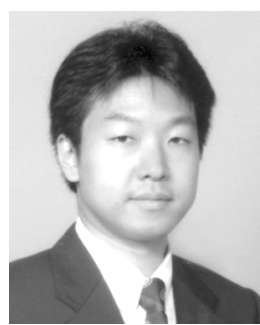

Takayuki Arai (S'90-M'91-SM'04) received the B.E., M.E., and Ph.D. degrees in electrical and electronics engineering from Sophia University, Tokyo, Japan, in 1989, 1991, and 1994, respectively.

From 1992 to 1993 and 1995 to 1996, he was with the Oregon Graduate Institute of Science and Technology, Portland, OR. From 1997 to 1998, he was with the International Computer Science Institute, Berkeley, CA. He is currently Professor of the Department of Information and Communication Sciences, Sophia University. From 2003 to 2004, he is a Visiting Scientist at the Massachusetts Institute of Technology, Cambridge, MA. His research interests include signal processing, acoustics, speech and hearing sciences, and spoken language processing.

Prof. Arai is a Member of the International Speech Communication Association, the Institute of Electronics, Information, and Communication Engineers, the Acoustical Society of America, the Acoustical Society of Japan, etc.

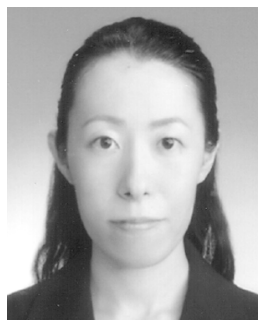

Nao Hodoshima (M'09) received the B.S., M.S., and $\mathrm{Ph} . \mathrm{D}$. degrees in electrical and electronics engineering from Sophia University, Tokyo, Japan, in 2002, 2004, and 2007, respectively.

She was a Visiting Researcher at the Department of Electronics and Telecommunications, Norwegian University of Science and Technology, Trondheim (2004-2005), a Research Fellow of the Japan Society for the Promotion of Science (2005-2007), a Research Associate at the Department of Electrical and Electronics Engineering, Sophia University, Tokyo (2007-2008), and a Postdoctoral Fellow at the Department of Information and Communication Sciences, Sophia University, Tokyo (2008-2010). She is currently a Lecturer at the Department of Information Media Technology, Tokai University, Tokyo. Her research interests are speech signal processing, hearing sciences, psychoacoustics, speech production, and room acoustics.

Dr. Hodoshima is a member of the International Speech Communication Association, the Institute of Electronics, Information and Communication Engineers, the Acoustical Society of America, and the Acoustical Society of Japan.

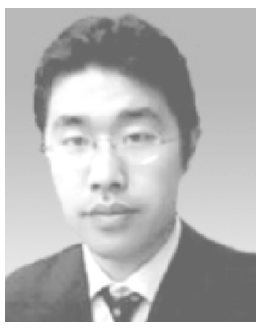

Keiichi Yasu (M'06) received the B.S. and M.S. degrees in electrical and electronics from Sophia University, Tokyo, Japan, in 2002 and 2004, respectively. $\mathrm{He}$ is currently pursuing the $\mathrm{Ph} . \mathrm{D}$. degree in the Graduate School of Science and Technology, Sophia University.

He was a research associate in the Department of Electrical and Electronics Engineering, Sophia University. He is currently a Research Assistant in the Graduate School of Science and Technology, Sophia University. He is interested in speech perception of elderly and hearing-impaired listeners.

Mr. Yasu is a member of The Institute of Electronics, Information and Communication Engineers, Japan, The Acoustical Society of Japan, and The Japan Society of Logopedics and Phoniatrics. He was honored to receive the student scholarship award at the International Hearing Aid Research Conference 2004. 